\title{
Inclusivity on all scales
}

\author{
With six months behind us, we would like to thank everyone who has submitted a paper, written for us or \\ refereed for us. We take this opportunity to clarify our policies and quell some enduring misconceptions.
}

So far this year, we have published a range of subjects, from dwarf planets, through large voids in the Local Group, to the most massive black holes in the Universe - and everything in between, including laboratory studies of meteorites and Moon rocks. And for any topic within our scope, we are happy to consider observational or theoretical studies, which may include advances in technique or computation. Indeed, our Mission Control series focuses on instrumentation at telescopes and/or on space missions.

This inclusive approach we apply to both research papers and the content in our magazine-style 'front half', where experts talk about policy, issues affecting the community (as well as our society in general) and the history of astronomy, to name but a few examples. We can even dedicate an entire issue to a particular theme, such as the Focus on gender equity in June.

And we are not confined to the Internet. Editors travel to conferences and institutions, sponsoring sessions or whole meetings even judging poster prizes. In fact, at the European Week of Astronomy and Space Science in Prague later this month, we will be hosting a luncheon to promote equity.

While we are out and about, we still sense some remaining confusion over our relationship with Nature. We are separate entities, to be sure. Nature will continue to publish papers in astronomy, but they are looking for the most significant advances, which people outside the field would want to know about. As we are more of a specialist journal, Nature Astronomy is able to accommodate papers that are narrower in scope. Moreover, being an online-only journal, we have more space and can consider topics that may be too technical or speculative for Nature.

The two journals are editorially independent - that is, we consider submitted papers against our own criteria and do not know which papers are under consideration at Nature. However, if Nature declines to publish a paper, the authors may choose to transfer their manuscript (along with any referees' reports, if appropriate) to us at the click of a button. There is no need to re-enter all the manuscript files and information, although submitting the manuscript without its 'history' remains an option. The same goes for Nature Physics and Nature Geoscience. As a group, we offer

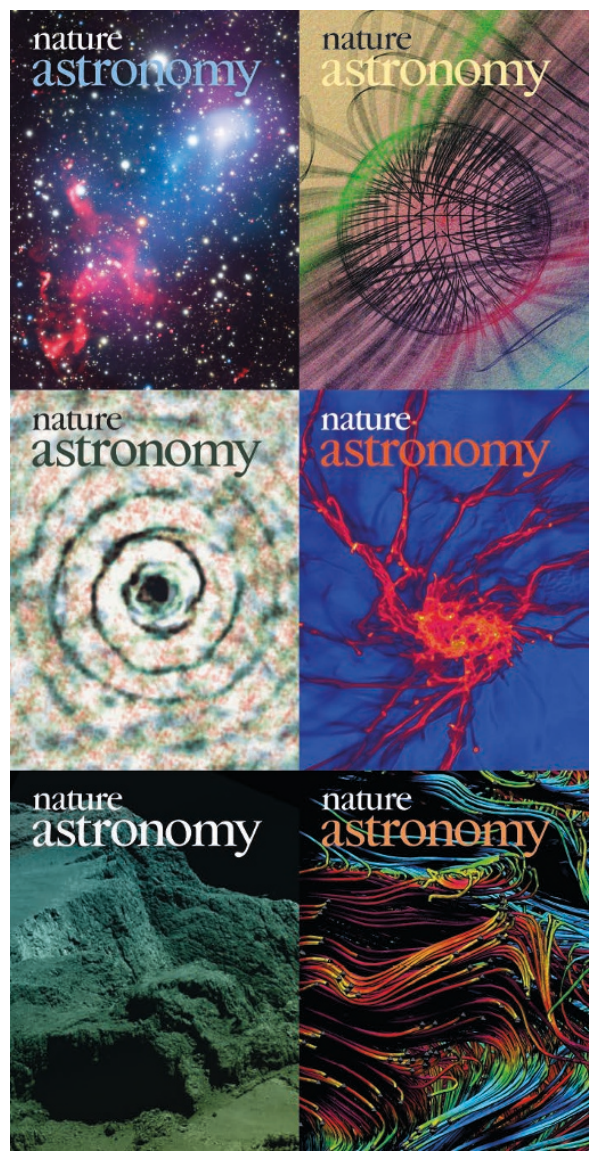

Image credits, clockwise from top-left: NASA/ CXC/SAO/R. van Weeren et al., NCRA/TIFR/ GMRT, NAOJ/Subaru; Daniel Pomarède (CEA Saclay); Enrico Corsaro (INAF OACT) \& Yueh-Ning Lee (CEA Saclay); Tamara Rogers (Newcastle University); Maurizio Pajola et al., ESA/Rosetta/MPS/OSIRIS MPS/UPD/LAM/ IAA/SSO/INTA/UPM/DASP/IDA; ALMA (ESO/ NAOJ/NRAO), Hyosun Kim et al.

astronomers, astrophysicists and planetary scientists a choice of platform to engage with the community they want to address.

Besides questions on the plurality of journals, we also tend to hear about a few enduring misconceptions. Many authors, readers and journalists alike maintain that we don't allow postings of preprints, that we don't allow authors to talk about their work under consideration at conferences, and that we put everything behind a paywall so that authors cannot share their work with others who don't have access. All FAKE NEWS!
As official company policy, we have been open about preprint archives since 1997. Authors are welcome to post their preprints at any stage of the submission process. The authors' final submitted version, which is not typeset or edited by us, should be posted to their institutions' repositories or updated on the preprint archive six months after publication. We just ask that the official published version, which we have helped to improve through layers of typesetting and editing, not be posted online. Instead, we encourage authors to share their published work through our SharedIt initiative (http:// www.springernature.com/gp/researchers/ sharedit). Every published article has a SharedIt link that can be sent to colleagues and posted on social media. This link is to a read-only PDF that can be annotated and shared with anyone, and it is a free service.

As for conferences, we encourage people to present their work to their peers. Our embargo means that papers under consideration should not be discussed with journalists until the week before publication, when we alert the media. The embargo ensures that stories that hit the news are peer reviewed, and it gives all media outlets the same length of time to prepare their stories and talk to authors and experts. Thus we do not gag authors at all. To the contrary, we are happy to shout about the papers that we publish - when the time is ripe.

And finally, authors often ask about formatting. Our response has always been that we can read a paper in almost any format. We put a lot of work into the final file, but at submission we are flexible as long as the manuscript provides a coherent narrative with enough detail for the referees to assess the robustness of the results. The more technical details can go into the Methods section so as not to interrupt the flow of the main text. But as we have a broad audience, please remember that context is very important. It is useful to provide some background so that the actual advance is clear. If you are not sure whether your work is suitable, we are happy to provide feedback before you even write it up. These 'presubmission' enquiries can be done formally through our website at mts-natastron.nature.com or by e-mail at natureastro@nature.com. A short summary is usually enough. Any other comments or suggestions are also welcome. 